\title{
Immediate effects of physical therapy on postural instability and frontal lobe dysfunction, as indicated by Frontal Assessment Battery score, in Parkinson's disease
}

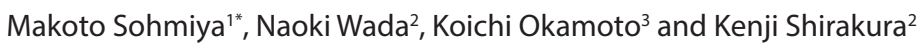

*Correspondence: soumiya@paz.ac.jp

\author{
CrossMark \\ $\leftarrow$ Click for updates
}

\begin{abstract}
'Graduate School of Health Sciences, Gunma PAZ University, Takasaki, Gunma, Japan. ${ }^{2}$ Department of Rehabilitation Medicine, Gunma University Graduate School of Medicine, Maebashi, Gunma, Japan. ${ }^{3}$ Department of Neurology, Geriatrics Research Institute and Hospital, Maebashi, Gunma, Japan.
\end{abstract}

\begin{abstract}
Background: The association between the immediate effects of physical therapy on motor symptoms and frontal lobe dysfunction has not been clarified in patients with Parkinson's disease. This study examined the immediate effects of physical therapy on postural instability in patients with Parkinson's disease and whether the improvement in postural instability was associated with Frontal Assessment Battery (FAB) score, as an indicator of frontal lobe dysfunction.

Methods: Twelve patients with idiopathic Parkinson's disease (Hoehn-Yahr classification range 3-4), independent ambulation, and no dementia were divided into FAB high-score (score $\geq 13, \mathrm{n}=6$ ) and lowscore (score $\leq 12, \mathrm{n}=6$ ) groups. Postural parameter data was acquired using a three-dimensional motion analysis system and a three-dimensional inclination and horizontal stimulation system before and after a 30-min physical therapy program. Measurements were obtained for total displacement of the center of gravity (COG), total anterior-posterior (AP) displacement of the spinous process of the 7 th cervical vertebra (C7) marker, maximum AP displacement of the $\mathrm{C} 7$ marker, maximum anterior speed of the $\mathrm{C} 7$ marker, and maximum posterior speed of the $\mathrm{C} 7$ marker.

Results: The high-score group showed significant decreases in total displacement of the COG and total AP displacement, maximum AP displacement, maximum anterior speed, and maximum posterior speed of the $\mathrm{C} 7$ marker. The low-score group showed no significant changes. FAB score was significantly correlated with change in maximum AP displacement of the $\mathrm{C} 7$ marker. Multivariate logistic regression analysis showed FAB score was the only predictor of improvement in total AP displacement and maximum AP displacement of the $\mathrm{C} 7$ marker.

Conclusions: There may be an association between the immediate effects of physical therapy on PI and FAB score in Parkinson's disease. Thus, FAB score could be useful for predicting which the patients with Parkinson's disease would be more likely to show the immediate effects of physical therapy on postural instability.

Keywords: Frontal Assessment Battery, immediate effects, Parkinson’s disease, physical therapy, threedimensional motion analysis, three-dimensional inclination and horizontal stimulation system, postural instability
\end{abstract}

\section{Introduction}

Postural instability (PI) and gait disturbance are common symp- toms in the late stage of Parkinson's disease (PD), where PI is due to the loss of postural reflexes. Although not common in 
the early stages of the disease, $\mathrm{Pl}$ is one of the most common factors that cause distress in the later stages [1]. PI, alongside gait disturbance, hypokinesia, and rigidity, can result in falls, fractures, and fear of fall and ultimately to reductions in daily activities and functional independence [2].

As PD progresses, various complex non-motor symptoms [3] tend to occur more often. Among them, frontal lobe dysfunction (FLD), which is associated with motor learning and executive disorder, modifies the motor symptoms. The presence and severity of dysexecutive syndrome can be assessed using the Frontal Assessment Battery (FAB), a simple bedside battery that evaluates such skills as motor programming, inhibitory control, and environmental autonomy [4].

The efficacy of physical activity such as physical therapy and treadmill training has been reported for PD [5-7]. The effects of physical therapy can be shown by the analysis of detailed kinematics data to determine PI by, for example, using a three-dimensional motion analysis system (3D-MAS) and a three-dimensional inclination and horizontal stimulation system (3D-IHSS). We previously reported an association between the immediate effects of physical therapy on gait disturbance and FAB score [8], but there have been no reports to date on whether there is an association between the immediate effects of physical therapy on PI and FAB score, as a potential predictor of improvements in PI.

The aim of this study was to investigate whether the immediate effects of physical therapy on PI in PD patients was associated with FAB score by employing 3D-MAS and 3D-IHSS, and $\mathrm{FAB}$ score could be a predictor of improvement in PI by using multivariate logistic regression analysis.

\section{Methods}

The study was approved by the Ethics Committee of Gunma University Hospital, Japan and all participants provided written consent before the study commenced.

\section{Participants}

Twelve PD outpatients with PI at our hospital were recruited consecutively for this study. In line with the methods of our previous study evaluating the immediate effects of physical therapy on gait disturbance and on FAB score [8], a diagnosis of idiopathic PD was confirmed in all participants by general and neurological examination conducted according to the UK Brain Bank criteria by two neurologists with a special interest in movement disorders [9]. All participants were classified as Hoehn-Yahr 3-4. All were receiving stable pharmacological treatment, with the total dose of antiparkinsonian medication calculated as levodopa equivalent dose (LED) [10]. As in our previous study [8], participants simultaneously underwent the following evaluations before kinematics measurement: Unified PD Rating Scale (UPDRS) assessment [11], Mini-Mental State Examination (MMSE) [12], and FAB. To exclude other diseases except $P D$, magnetic resonance imaging (MRI) and technetium-99m ethyl cysteinate dimer single photon emis- sion computed tomography (ECD-SPECT) were performed. ECD-SPECT was evaluated using an easy Z-score imaging system (eZIS) and quantitative analysis of global and regional cerebral blood flow (CBF) in 14 regions of interest [13].

Exclusion criteria were as follows; (1) Patient had any other neuromuscular, cardiopulmonary, osteoarticular or psychiatric disorders. (2) Patient could not walk independently without an assistive device such as a cane or walker. (3) Medication for PD was modified within one month prior to the study. (4) Patient had manifested the on-off phenomenon. (5) Patient had participated in PT or any rehabilitation program in the previous 2 weeks. (6) Patient with an MMSE score $\leq 25$. (7) Patient with specific abnormalities except for mild atrophic change by MRI or significant difference in global or regional CBF by ECD-SPECT (Table 1).

\section{FAB}

FAB score correlates with scores on other evaluation tools, namely, the Wisconsin card sorting test and symbol search [14]. The FAB domains of conceptualization, mental flexibility, motor programming, sensitivity to interference, inhibitory control, and environmental autonomy are graded using a 4-point scale (0-3) for a total score of 18 [4]. Patient with frontal lobe dysfunction has a low FAB score [4]. Based on the median $F A B$ score of 13 , patients were assigned to a high-score group $(n=6$; score: $\geq 13)$ or a low-score group $(n=6$; score: $\leq 12)$.

\section{Physical therapy session}

One 30-min physical therapy protocol was administered by experienced, licensed physical therapists (Table 2).

\section{Trunk movement analysis}

A combined system comprised of 10-camera 3D-MAS (Vicon612 $2^{\circ}$, Oxford Metrics, Oxford, UK) and 3D-IHSS (GS-6900B', Anima Corp, Tokyo, Japan) was used to acquire kinematic data on trunk movement before and after the physical therapy session. Analysis of postural movement was performed at the university hospital before and after the intervention.

3D-IHSS can measure the center of gravity (COG) and has the platform that can give stimulations in an anterior-posterior or oblique direction (Figure 1). The system also can set the protocol of platform movement (movement distance, duration of a cycle, frequency). While giving patients the stimulations on the moveable platform, 3D-IHSS collects the data of the COG. The data is total displacement of the COG. Mean values of 3 trials were calculated for each examination and were used for analysis.

The test-retest reliability of total COG displacement provided by 3D-IHSS has been investigated. The reliability coefficients before and after intervention were 0.811 and 0.857 , respectively.

All patients were asked to stand without any standing aids on the 3D-IHSS moveable platform in the rehabilitation unit. A safety harness was fitted to avoid falls. 3D-IHSS provided 
Sohmiya et al, Physical Therapy and Rehabilitation 2018, http://www.hoajonline.com/journals/pdf/2055-2386-5-10.pdf

Table 1. Clinical characteristics of patients with Parkinson's disease and FAB scores.

\begin{tabular}{|c|c|c|c|c|}
\hline & \\
\hline & Total & FAB high-score group & FAB low-score group & p value \\
\hline & $(\mathrm{N}=12)$ & $(\mathrm{n}=6)$ & $(\mathrm{n}=6)$ & \\
\hline Males / Females (n) & $5 / 7$ & $2 / 4$ & $3 / 3$ & n.s.* \\
\hline Age (years) & $70.1 \pm 5.5$ & $69.0 \pm 6.8$ & $71.2 \pm 3.3$ & n.s.** \\
\hline Body height (cm) & $156.3 \pm 8.5$ & $157.5 \pm 9.8$ & $155.2 \pm 6.9$ & n.s.** \\
\hline Body weight $(\mathrm{kg})$ & $52.7 \pm 9.2$ & $53.7 \pm 11.6$ & $51.7 \pm 5.8$ & n.s.** \\
\hline Duration of illness (year) & $10.5 \pm 6.8$ & $7.5 \pm 3.3$ & $13.5 \pm 8.1$ & n.s.** \\
\hline Yahr classification (Hoehn-Yahr 3/4) & $10 / 2$ & $6 / 0$ & $4 / 2$ & n.s.* \\
\hline \multicolumn{5}{|l|}{ Anti-parkinsonian medication (mg/day) } \\
\hline LED & $610.8 \pm 210.8$ & $624.8 \pm 197.1$ & $597.9 \pm 223.7$ & n.s.** \\
\hline L-dopa / decarboxylase inhibitor & $366.7 \pm 119.6(12$ cases $)$ & $358.3 \pm 148.4$ ( 6 cases $)$ & $375.0 \pm 80.4$ ( 6 cases $)$ & n.s.** \\
\hline Pergolide mesilate & $0.63 \pm 0.01$ ( 5 cases $)$ & $0.75 \pm 0.02$ ( 2 cases $)$ & $0.54 \pm 0.01$ ( 3 cases) & n.s.** \\
\hline Cabergoline & $2.3 \pm 1.0(7$ cases $)$ & $2.7 \pm 0.9(3$ cases $)$ & $2.2 \pm 1.3(4$ cases $)$ & n.s.** \\
\hline Bromocriptine mesilate & $2.0 \pm 0.0$ ( 1 cases $)$ & $2.0 \pm 0.0$ ( 1 cases $)$ & $0.0 \pm 0.0$ ( 0 cases $)$ & n.s.** \\
\hline Amantadine hydrochloride & $131.3 \pm 34.8$ ( 8 cases $)$ & $150.0 \pm 0.0$ ( 4 cases $)$ & $112.5 \pm 41.5$ ( 4 cases $)$ & n.s.** \\
\hline Deprenyl & $4.4 \pm 2.1$ ( 8 cases $)$ & $5.0 \pm 2.5(4$ cases $)$ & $3.8 \pm 1.3$ ( 4 cases) & n.s.** \\
\hline Trihexyphenidyl hydrochloride & $4.0 \pm 2.0$ ( 2 cases $)$ & $6.0 \pm 0.0(1$ cases $)$ & $2.0 \pm 0.0$ ( 1 cases $)$ & n.s.** \\
\hline \multicolumn{5}{|l|}{ UPDRS } \\
\hline Part 1 & $1.5 \pm 1.3$ & $1.8 \pm 1.1$ & $1.2 \pm 1.3$ & n.s.** \\
\hline Part 2 & $11.7 \pm 6.9$ & $9.0 \pm 3.9$ & $14.3 \pm 8.2$ & n.s.** \\
\hline Part 3 & $21.6 \pm 9.6$ & $21.3 \pm 10.4$ & $21.8 \pm 8.6$ & n.s.** \\
\hline Part 4 & $2.4 \pm 2.3$ & $1.8 \pm 1.3$ & $3.0 \pm 2.8$ & n.s.** \\
\hline Total MMSE score & $28.5 \pm 1.8$ & $28.8 \pm 1.9$ & $28.2 \pm 1.7$ & n.s.** \\
\hline \multicolumn{5}{|l|}{ FAB score } \\
\hline Conceptualization & $1.6 \pm 1.0$ & $1.7 \pm 0.7$ & $1.5 \pm 1.3$ & n.s. *** \\
\hline Mental Flexibility & $2.2 \pm 0.8$ & $2.2 \pm 0.7$ & $2.2 \pm 0.9$ & n.s. ** \\
\hline Motor programming & $2.8 \pm 0.6$ & $3.0 \pm 0.0$ & $2.5 \pm 0.8$ & n.s. *** \\
\hline Sensitivity to interference & $2.8 \pm 0.4$ & $3.0 \pm 0.0$ & $2.5 \pm 0.5$ & n.s. *** \\
\hline Inhibitory control & $1.5 \pm 1.1$ & $2.3 \pm 0.5$ & $0.7 \pm 0.9$ & $<0.01^{* *}$ \\
\hline Environmental autonomy & $2.5 \pm 0.5$ & $2.8 \pm 0.4$ & $2.2 \pm 0.4$ & $<0.05 * *$ \\
\hline Total & $13.3 \pm 2.2$ & $15.0 \pm 1.4$ & $11.5 \pm 1.4$ & $<0.005^{* * *}$ \\
\hline
\end{tabular}

FAB: Frontal Assessment Battery, LED: levodopa equivalent dose, UPDRS: Unified Parkinson's disease Rating Scale, MMSE: Mini-Mental State Examination, Mean \pm SD, n.s. $=$ not significant, statistical analysis: ${ }^{*}$ Chi-square test, ${ }^{* *}$ Mann-Whitney Utest.

Table 2. Physical therapy protocol.

(1) Stretching exercises $(5 \mathrm{~min})$ Exercises mainly targeting the trunk and lower extremities, especially the ankle joint.

(2) Strengthening exercises $(5 \mathrm{~min})$ Exercises mainly targeting hip flexor and knee extensor muscles. Low intensity (20-30 repetitions maximum) isokinetic exercises were chosen.

(3) Balance training (5 min) Maintaining balance on a soft mattress in a standing position.

While in the quadruped position, extending one upper limb together with contralateral lower limb.

(4) Recreational game played with a ball (5 min) Playing catch with the therapist using balls of different sizes and weights while sitting and standing.

(5) Gait training with external auditory cueing $(10 \mathrm{~min}) \quad$ Walking in time to music or a metronome (120 beats per minute).

stimulation in an anterior-posterior direction (movement distance, $10 \mathrm{~cm}$; duration of 1 anterior-posterior cycle, $2 \mathrm{~s}$; 6 cycles), and the combined 3D-MAS and 3D-IHSS system simultaneously acquired kinematic data on trunk movement.

A spherical retro-reflective surface marker was fixed to the patient's skin over the spinous process of the 7th cervical vertebra (C7). To ensure accuracy, 3D-MAS was calibrated prior to data collection. Using C7 as a positional marker [8], 3D-MAS measured the following parameters on the horizontal plane: total anterior-posterior (AP) displacement of the C7 marker, maximum AP displacement of the marker, maximum anterior speed of the marker, and maximum posterior speed of the marker. Mean values of 3 trials were calculated for each ex- amination and were used for analysis. All data were acquired by an assessor blinded to patient data.

We previously reported the reliability and accuracy of this system for recording three-dimensional kinematic data [8]. Interclass correlation coefficients ranged from 0.870 to 0.994 for intra-rater reliability and from 0.906 to 0.999 for inter-rater reliability.

\section{Statistical analysis}

Statistical analysis was performed using SPSS software Version 25.0 (IBM, Chicago, IL). As in our previous study [8], significant differences between the FAB high- and low-score groups were determined using the Mann-Whitney $U$ test or 


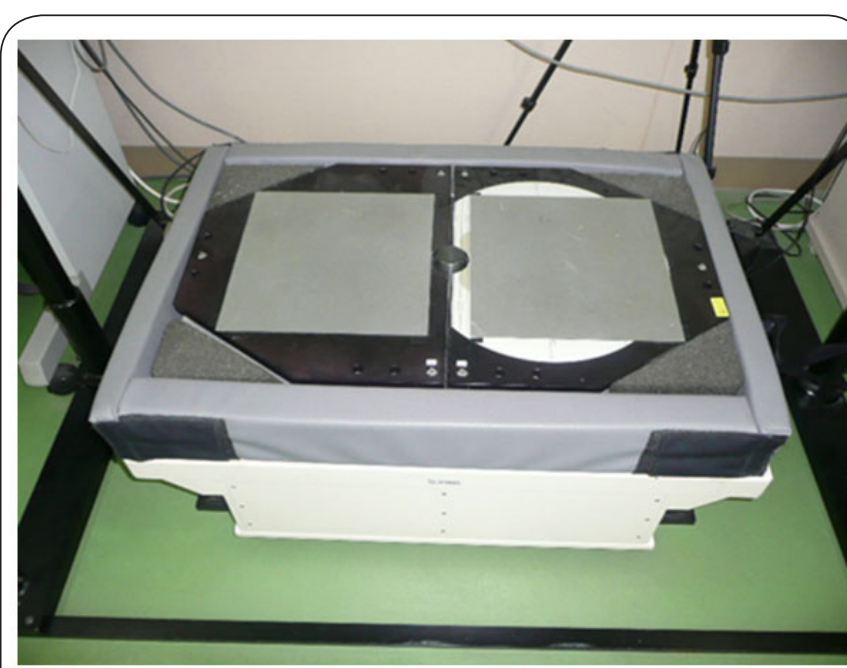

Figure 1. Three-dimensional inclination and horizontal stimulation system (3D-IHSS).

Chi-squared test. Pre- and post-measurements were compared with Wilcoxon's matched-pair signed-rank test. Correlations between variables were evaluated using Spearman's rank-order correlation coefficients. To predict the immediate effects of physical therapy, a multivariable logistic regression model controlling for possible confounding covariates was fitted by stepwise forward selection of variables: age, duration of illness, Hoehn-Yahr classification, LED, all four parts of the UPDRS, total MMSE score, and FAB score. Variables that were statistically significant were then included in multivariate logistic regression analysis. Because of the sample size and number of variables, the entry probability for logistic analysis was set at the 0.10 level of significance rather than the 0.05 level in an effort to avoid type II error. Associations between the clinical characteristics and the independent variables were determined by odds ratios (OR) with $95 \%$ confidence intervals (Cl). Statistical significance was set at $P<0.05$. Descriptive statistics are reported as mean \pm SD.

\section{Results}

Comparisons between the FAB high-and low-score groups before physical therapy intervention revealed no significant differences in sex, age, duration of illness, Hoehn-Yahr classification, antiparkinsonian medication, LED, all four parts of the UPDRS, or total MMSE score. The FAB low-score group showed significant decreases in inhibitory control, environmental autonomy, and total scores compared with the high-score group (Table 1). MRI revealed no specific abnormalities except for mild atrophic change. Quantitative analysis by ECD-SPECT showed no significant difference in global or regional CBF between the two groups.

Comparisons before and after physical therapy revealed the following results (Table 3). The high-score group showed significant decreases in total displacement of the COG, total AP displacement of the $\mathrm{C} 7$ marker, maximum AP displacement of the marker, maximum anterior speed of the marker, and maximum posterior speed of the marker. The low-score group showed no significant differences. FAB score correlated significantly with changes in maximum AP displacement of the $\mathrm{C} 7$ marker and maximum anterior speed of the $\mathrm{C} 7$ marker (Table 4, Figure 2).

Multivariate logistic regression analysis revealed that $F A B$ score was the only predictor of improvement in total AP displacement of the $\mathrm{C} 7$ marker and maximum AP displacement of the $C 7$ marker. In addition to FAB score, analysis showed MMSE score, UPDRS part 3 (which involves the evaluation of

Table 3. Changes in postural stability parameters between before and after physical therapy.

\begin{tabular}{lll}
\hline $\begin{array}{l}\text { FAB high-score group } \\
(\mathrm{n}=6)\end{array}$ & $\mathrm{p}$ value & $\begin{array}{l}\text { FAB low-score group } \\
(\mathrm{n}=6)\end{array}$ \\
\hline
\end{tabular}

Total displacement of COG $(\mathrm{cm})$

$\begin{array}{llll}58.4 \pm 83.7 & <0.05 & 5.0 \pm 28.5 & \text { n.s. }\end{array}$

Total AP displacement of C7 marker $(\mathrm{cm})$

$158.5 \pm 299.3$

Maximum AP displacement of C7 marker (cm)

$17.2 \pm 19.0$

Maximum anterior speed of $\mathrm{C} 7$ marker $(\mathrm{cm} / \mathrm{s})$

$$
23.8 \pm 40.1
$$

Maximum posterior speed of $\mathrm{C} 7$ marker $(\mathrm{cm} / \mathrm{s})$

$$
32.5 \pm 42.1
$$

$$
<0.05
$$

$5.0 \pm 28.5$

$4.2 \pm 98.0$

$-4.0 \pm 24.8$

$-6.8 \pm 37.2$

$17.5 \pm 44.7$ n.s.

n.s.

n.s.

n.s.

n.s

FAB: Frontal Assessment Battery, COG: Center of gravity, AP: anterior-posterior, C7: spinous process of the 7th cervical vertebra Mean \pm SD, n.s.: not significant, statistical analysis: Wilcoxon matched pairs signed rank Test 
Table 4. Correlation between FAB score and changes in postural stability parameters between before and after physical therapy.

\begin{tabular}{lcc} 
& Correlation coefficient & p value \\
\cline { 2 - 3 } Total displacement of COG $(\mathrm{cm})$ & 0.094 & n.s. \\
Total AP displacement of C7 marker $(\mathrm{cm})$ & 0.305 & n.s. \\
Maximum AP displacement of C7 marker $(\mathrm{cm})$ & 0.462 & $<0.01$ \\
Maximum anterior speed of C7 marker $(\mathrm{cm} / \mathrm{s})$ & 0.390 & $<0.05$ \\
Maximum posterior speed of C7 marker $(\mathrm{cm} / \mathrm{s})$ & 0.268 & n.s.
\end{tabular}

FAB: Frontal Assessment Battery, COG: Center of gravity, AP: anterior-posterior, C7: spinous process of the 7th cervical vertebra Mean \pm SD, n.s.: not significant, statistical analysis: Spearman's rank order correlation coefficient.

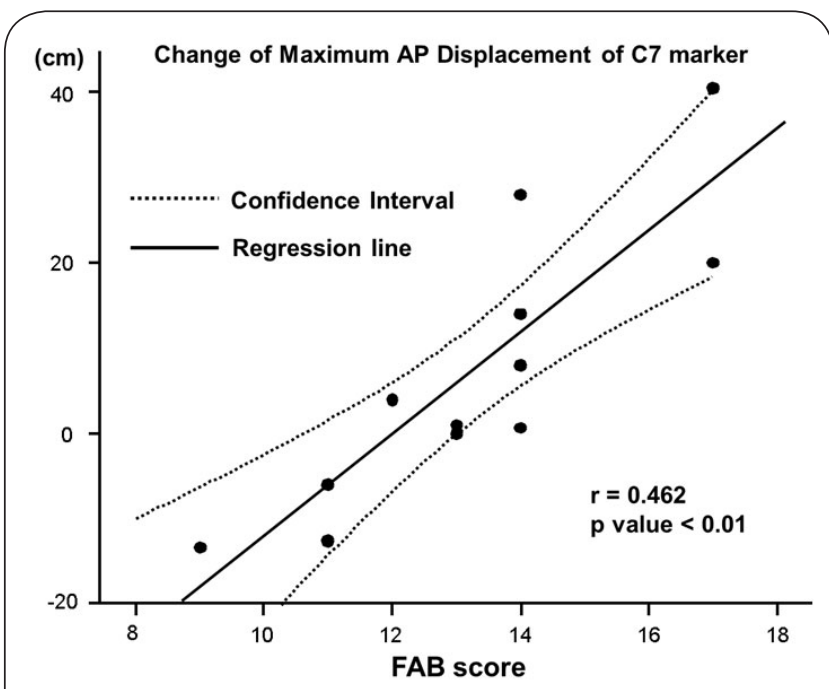

Figure 2. Frontal Assessment Battery score and change in maximum anterior-posterior displacement of the marker of the spinous process of the 7 th cervical vertebra.

AP: anterior-posterior, C7: spinous process of the 7th cervical vertebra, statistical analysis: Spearman's rank order correlation coefficient.

posture and postural stability), and LED were predictors of improvement in total displacement of the COG and maximum anterior and posterior speed of the C7 marker. No other significant relationships were observed between clinical characteristics and improved PI (Table 5).

\section{Discussion}

\section{PD and the immediate effects of Physical therapy}

Through improvements in gait, postural stability, and muscle power, physical capacity has been shown to be improved in PD with the use of cueing strategies in long-term physical therapy and home training [5-7,15]. Moreover, treadmill training sessions have been shown to immediately improve gait in PD $[16,17]$. It is important, however, to clarify not only the long- term effects but also the immediate effects of physical therapy because it may immediately improve physical capabilities and thus help to prevent falls [18]. Therefore, to investigate whether physical therapy intervention could immediately improve $\mathrm{PI}$ in PD patients, our physical therapy session consisted of intervention activities that are reported to be effective for PI improvement, namely, stretching exercises, balance training, and gait training with external auditory cueing [19].

\section{FLD and motor symptoms}

FLD, including executive dysfunction, is the main feature of non-motor symptoms in PD [3]. Non-motor symptoms are closely connected with the effects of physical therapy on motor symptoms. A physical exercise program was reported to improve executive function in PD patients [20], and accordingly in this study we focused especially on the immediate effects of physical therapy on PI and FLD.

\section{FAB score and FLD}

We decided to use FAB as a convenient evaluation tool for FLD in this study. FAB is a short, simple instrument consisting of six subtests that explore different abilities related to frontal lobe functions. Its results correlate with those of other commonly performed evaluation tools for PD, namely, the Wisconsin card sorting test and symbol search, the standardized tests for the assessing executive function [14].

\section{FAB score and the immediate effects of physical therapy on PI}

In this study, we wanted to address the question of whether a physical therapy session could immediately improve PI (confirmed kinematically) and whether FAB score could reflect the improvement in PI. Using detailed kinematics data recorded using both 3D-MAS and 3D-IHSS, we could accurately detect post-intervention alterations in not only displacement of the COG, but also C7 displacement and speed. We were then able to determine, for the first time, an association between these post-intervention changes in postural parameters and $F A B$ 
Sohmiya et al, Physical Therapy and Rehabilitation 2018,

http://www.hoajonline.com/journals/pdf/2055-2386-5-10.pdf

doi: $10.7243 / 2055-2386-5-10$

Table 5. Stepwise logistic model for predicting the immediate effects of physical therapy on postural instability.

\begin{tabular}{|c|c|c|c|c|c|c|c|c|}
\hline & \multicolumn{2}{|c|}{ FAB score } & \multicolumn{2}{|c|}{ MMSE score } & \multicolumn{2}{|c|}{ UPDRS part 3 score } & \multicolumn{2}{|l|}{ LED } \\
\hline & $p$ value & $\mathrm{OR}(95 \% \mathrm{CI})$ & $p$ value & $\mathrm{OR}(95 \% \mathrm{CI})$ & $p$ value & OR $(95 \% \mathrm{CI})$ & $p$ value & $\mathrm{OR}(95 \% \mathrm{CI})$ \\
\hline \multicolumn{9}{|l|}{ Total displacement of COG } \\
\hline & n.s. & & n.s. & & 0.026 & $\begin{array}{l}1.046 \\
(0.903-1.212)\end{array}$ & n.s. & \\
\hline \multicolumn{9}{|c|}{ Total AP displacement of $\mathrm{C} 7$ marker } \\
\hline & 0.045 & $\begin{array}{l}1.268 \\
(0.708-2.270)\end{array}$ & n.s. & & n.s. & & n.s. & \\
\hline \multicolumn{9}{|c|}{ Maximum AP displacement of C7 marker } \\
\hline & 0.0002 & $\begin{array}{l}5.713 \\
(0.801-40.758)\end{array}$ & n.s. & & n.s. & & n.s. & \\
\hline \multicolumn{9}{|c|}{ Maximum anterior speed of $\mathrm{C} 7$ marker } \\
\hline & 0.044 & $\begin{array}{l}1.445 \\
(0.525-3.978)\end{array}$ & 0.010 & $\begin{array}{l}2.077 \\
(0.806-5.353)\end{array}$ & n.s. & & 0.043 & $\begin{array}{l}1.005 \\
(0.996-1.015)\end{array}$ \\
\hline \multicolumn{9}{|c|}{ Maximum posterior speed of $\mathrm{C} 7$ marker } \\
\hline & n.s. & & 0.017 & $\begin{array}{l}1.385 \\
(0.699-2.742)\end{array}$ & n.s. & & n.s. & \\
\hline
\end{tabular}

FAB: Frontal Assessment Battery, MMSE: Mini-Mental State Examination, UPDRS: Unified PD Rating Scale, LED:

levodopa equivalent dose, COG: Center of gravity, AP: anterior-posterior, C7: spinous process of the 7th cervical vertebra,

OR: odds ratio, CI: 95\% confidence interval, n.s.: not significant

score. Specifically, the FAB high-score group showed several immediate improvements in trunk movement: decreased changes in total displacement of the COG and in total AP displacement, maximum AP displacement, maximum anterior speed, and maximum posterior speed of the C7 marker. The low-score group showed no significant changes. Moreover, FAB score correlated positively with the changes in maximum AP displacement and maximum anterior speed of the $\mathrm{C} 7$ marker. Thus, the present study shows an association between the immediate effects of physical therapy on PI and FAB score. Multivariate logistic regression analysis then revealed that FAB score was a predictor of improvement in PI. Thus, FAB score could be useful for predicting the immediate effects of physical therapy on PI in PD.

FAB domain scores and the immediate effects of physical therapy on PI

The FAB low-score group showed significantly lower scores in the domains of inhibitory control and environmental autonomy. Inhibitory control assesses control of impulsiveness and withholding of a response to external stimuli through practice, while environmental autonomy assesses the spontaneous tendency to adhere to the environment through prehension behavior [4]. Both skills are essential for postural stability and avoiding falls caused by external stimuli. Kataoka et al found a significantly lower score for inhibitory control among fallers with PD than in non-fallers with PD [21]. Deficits in environmental autonomy mean that PD patients are more susceptible to external stimuli, which makes avoiding falls difficult.
In the present study, the FAB low-score group also showed a lower score for motor programming compared with the highscore group, albeit not significantly lower. This finding reflects a process of improved motor performance through practice during physical therapy, and has previously been associated with the positive effects of rehabilitation [22].

Our FAB results therefore show an association between the immediate effects of physical therapy on PI and FLD with FAB domain scores. Meanwhile, we should note that the correlation coefficients were not high (Table 4, Figure 2). It means most of the variance between variables cannot be explained. We intend to solve the problem by increasing the number of subjects in future research.

It is also important to note that our findings do not suggest physical therapy is ineffective for PD patients with a low FAB score because only PI was investigated and the aims and content of physical therapy interventions are numerous and diverse.

Frontal lobe connections and immediate effects of physical therapy on PI

King et al hypothesized that frontal lobe connections with the basal ganglia and brainstem posture and locomotor centers are responsible for postural deficits in PD patients and play a role in rehabilitation efficacy [23]. The immediate effects of physical therapy on PI can be seen when damage to these connections is mild, and progressive damage to these connections may decrease the immediate effects. So, a decline in frontal lobe function with the progression PD may affect the immediate effects. Because it is difficult to predict the imme- 
Sohmiya et al, Physical Therapy and Rehabilitation 2018,

diate effects of physical therapy by neurological examination and use of evaluation scales (e.g., UPDRS), FAB score could be a useful predictor of which patients could benefit most from the immediate effects of PT.

\section{Other predictors and the immediate effects of physical therapy}

Of note, multivariate logistic regression also indicated that MMSE score, UPDRS part 3 score, and LED were predictors of the immediate effects of physical therapy. MMSE is the basic examination for cognitive impairment. Although no significant difference was noted between the FAB high- and low-score groups in the present study, cognitive impairment develops as PD progresses. UPDRS part 3 involves motor examination and includes evaluations of posture and postural stability [11]. It is not surprising that MMSE and UPDRS part 3 were identified as predictors. Moreover, it has been reported that antiparkinsonian medication, particularly L-dopa, dopamine agonists, and anticholinergic drugs can affect frontal function [24]. Although differences in LED doses were not found to be significant between the groups in the present study, they were higher in the high-score group than in the low-score group (Table 1).

\section{Limitations}

Some limitations of this study should be noted. First, the number of subjects was small, which limits the generalization of the results. Second, we used FAB alone to evaluate FLD, and further studies should use additional evaluation tools to verify our results. In addition, the test-retest reliability of FAB has not been investigated for PD patients. We should examine the reliability in the future. Going forward, the long-term effects after several physical therapy sessions should be compared with the immediate effects of a single session investigated here, and other disturbances aside from postural instability should be investigated. We intend to address these limitations in future research.

\section{Conclusion}

We demonstrated an association between the immediate effects of physical therapy on PI and FLD in PD. Our findings suggest that $F A B$ scores could be useful for predicting which PD patients would be more likely to show the immediate effects of PT on PI.

\section{Competing interests}

The authors declare that they have no competing interests.

\section{Acknowledgement}

This study was supported by a Grant-in-Aid for Scientific Research (No. 25350592, No. 17K01465) from the Japanese Ministry of Education, Culture, Sports, Science and Technology.
Authors' contributions

\begin{tabular}{|l|c|c|c|c|}
\hline Authors' contributions & MS & NW & KO & KS \\
\hline Research concept and design & $\checkmark$ & $\checkmark$ & -- & -- \\
\hline Collection and/or assembly of data & $\checkmark$ & $\checkmark$ & $\checkmark$ & -- \\
\hline Data analysis and interpretation & $\checkmark$ & -- & -- & -- \\
\hline Writing the article & $\checkmark$ & -- & -- & -- \\
\hline Critical revision of the article & $\checkmark$ & -- & -- & -- \\
\hline Final approval of article & $\checkmark$ & $\checkmark$ & $\checkmark$ & $\checkmark$ \\
\hline Statistical analysis & $\checkmark$ & -- & -- & -- \\
\hline
\end{tabular}

Publication history

Editor: Gordon John Alderink, Grand Valley State University, USA. Received: 06-May-2018 Final Revised: 25-Jun-2018

Accepted: 02-Jul-2018 Published: 24-Jul-2018

\section{References}

1. Kim SD, Allen NE, Canning CG and Fung VS. Postural instability in patients with Parkinson's disease. Epidemiology, pathophysiology and management. CNS Drugs. 2013; 27:97-112. | Article | PubMed

2. Grimbergen YA, Munneke M and Bloem BR. Falls in Parkinson's disease. Curr Opin Neurol. 2004; 17:405-15. I Article I PubMed

3. Pfeiffer RF. Non-motor symptoms in Parkinson's disease. Parkinsonism Relat Disord. 2016; 22 Suppl 1:S119-22. | Article | PubMed

4. Dubois B, Slachevsky A, Litvan I and Pillon B. The FAB: a Frontal Assessment Battery at bedside. Neurology. 2000; 55:1621-6. | Article | PubMed

5. Klamroth S, Steib S, Devan S and Pfeifer K. Effects of Exercise Therapy on Postural Instability in Parkinson Disease: A Meta-analysis. J Neurol Phys Ther. 2016; 40:3-14. | Article | PubMed

6. Goodwin VA, Richards SH, Taylor RS, Taylor AH and Campbell JL. The Effectiveness of exercise interventions for people with Parkinson's Disease: A systematic review and meta-analysis. Mov Disord. 2008; 23:631-40.

7. Morris $\mathrm{ME}$, lansek $\mathrm{R}$ and Kirkwood $\mathrm{B}$. A randomized controlled trial of movement strategies compared with exercise for people with Parkinson's disease. Mov Disord. 2009; 24:64-71. | Article I PubMed

8. Sohmiya M, Wada N, Tazawa M, Okamoto K and Shirakura K. Immediate effects of physical therapy on gait disturbance and frontal assessment battery in Parkinson's disease. Geriatr Gerontol Int. 2013; 13:630-7. | Article | PubMed

9. Hughes AJ, Daniel SE, Kilford L and Lees AJ. Accuracy of clinical diagnosis of idiopathic Parkinson's disease: a clinico-pathological study of $\mathbf{1 0 0}$ cases. J Neurol Neurosurg Psychiatry. 1992; 55:181-4. | $\underline{\text { Article | PubMed }}$ Abstract | PubMed FullText

10. Tomlinson CL, Stowe R, Patel S, Rick C, Gray R and Clarke CE. Systematic review of levodopa dose equivalency reporting in Parkinson's disease. Mov Disord. 2010; 25:2649-53. | Article | PubMed

11. Fahn S, Elton RL, and UPDRS program members. Unified Parkinsons Disease Rating Scale. In Fahn S, Marsden CD, Goldstein M, and Calne DB ( Eds.), Recent developments in Parkinsons disease. 1987.

12. Folstein MF, Folstein SE and McHugh PR. "Mini-mental state". A practical method for grading the cognitive state of patients for the clinician. J Psychiatr Res. 1975; 12:189-98. I Article I PubMed

13. Matsuda H, Mizumura S, Soma T and Takemura N. Conversion of brain SPECT images between different collimators and reconstruction processes for analysis using statistical parametric mapping. Nucl Med Commun. 2004; 25:67-74. | Article | PubMed

14. Lima CF, Meireles LP, Fonseca R, Castro SL and Garrett C. The Frontal Assessment Battery (FAB) in Parkinson's disease and correlations with formal measures of executive functioning. J Neurol. 2008; 255:1756-61. | Article | PubMed 
15. Capato TT, Tornai J, Avila P, Barbosa ER and Piemonte ME. Randomized controlled trial protocol: balance training with rhythmical cues to improve and maintain balance control in Parkinson's disease. $B M C$ Neurol. 2015; 15:162. | Article | PubMed Abstract | PubMed FullText

16. Frenkel-Toledo S, Giladi N, Peretz C, Herman T, Gruendlinger L and Hausdorff JM. Treadmill walking as an external pacemaker to improve gait rhythm and stability in Parkinson's disease. Mov Disord. 2005; 20:1109-14. | Article | PubMed

17. Pohl M, Rockstroh G, Ruckriem S, Mrass G and Mehrholz J. Immediate effects of speed-dependent treadmill training on gait parameters in early Parkinson's disease. Arch Phys Med Rehabil. 2003; 84:1760-6. | Article | PubMed

18. Sohmiya M, Wada N, Tazawa M, Okamoto K and Shirakura K. Immediate effects of physical therapy on gait disturbance and frontal assessment battery in Parkinson's disease. Geriatr Gerontol Int. 2013; 13:630-7. I Article | PubMed

19. Rochester L, Hetherington V, Jones D, Nieuwboer A, Willems AM, Kwakkel $G$ and Van Wegen $E$. The effect of external rhythmic cues (auditory and visual) on walking during a functional task in homes of people with Parkinson's disease. Arch Phys Med Rehabil. 2005; 86:999-1006. | Article I PubMed

20. Tanaka K, Quadros AC, Jr., Santos RF, Stella F, Gobbi LT and Gobbi S. Benefits of physical exercise on executive functions in older people with Parkinson's disease. Brain Cogn. 2009; 69:435-41. | Article | PubMed

21. Kataoka H, Tanaka N, Saeki K, Kiriyama T and Ueno S. Low frontal assessment battery score as a risk factor for falling in patients with Hoehn-Yahr Stage III Parkinson's disease: a 2-year prospective study. Eur Neurol. 2014; 71:187-92. | Article | PubMed

22. Nieuwboer $A$, Rochester L, Muncks $L$ and Swinnen SP. Motor learning in Parkinson's disease: limitations and potential for rehabilitation. Parkinsonism Relat Disord. 2009; 15 Suppl 3:S53-8. | Article | PubMed

23. King LA, Peterson DS, Mancini M, Carlson-Kuhta P, Fling BW, Smulders K, Nutt JG, Dale M, Carter J, Winters-Stone KM and Horak FB. Do cognitive measures and brain circuitry predict outcomes of exercise in Parkinson Disease: a randomized clinical trial. BMC Neurol. 2015; 15:218. | Article | PubMed Abstract | PubMed FullText

24. Cools R. Dopaminergic modulation of cognitive function-implications for L-DOPA treatment in Parkinson's disease. Neurosci Biobehav Rev. 2006; 30:1-23. | Article | PubMed

\section{Citation:}

Sohmiya M, Wada N, Okamoto K and Shirakura K. Immediate effects of physical therapy on postural instability and frontal lobe dysfunction, as indicated by Frontal Assessment Battery score, in Parkinson's disease. Phys Ther Rehabil. 2018; 5:10.

http://dx.doi.org/10.7243/2055-2386-5-10 